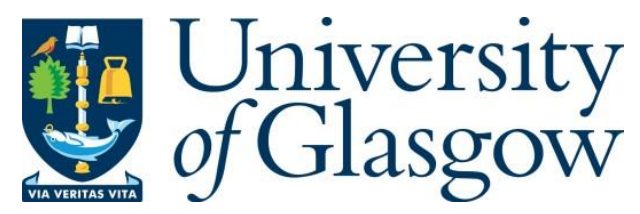

Saeed, A., Katranaras, E., Dianati, M., and Imran, M. A. (2016) Control and data channel resource allocation in OFDMA heterogeneous networks. Journal of Signal Processing Systems, 85(2), pp. 183-199.

There may be differences between this version and the published version. You are advised to consult the publisher's version if you wish to cite from it.

http://eprints.gla.ac.uk/132747/

Deposited on: 24 February 2017

Enlighten - Research publications by members of the University of Glasgow http://eprints.gla.ac.uk 


\title{
Control and Data Channel Resource Allocation in OFDMA Heterogeneous Networks
}

\author{
Arsalan Saeed, Efstathios Katranaras, Mehrdad Dianati, and Muhammad Ali Imran \\ Institute for Communication Systems (ICS) \\ University of Surrey, Guildford, United Kingdom \\ Email: \{arsalan.saeed, efstathios.katranaras, m.dianati, m.imran\}@surrey.ac.uk
}

\begin{abstract}
This paper investigates the downlink resource allocation problem in Orthogonal Frequency Division Multiple Access (OFDMA) Heterogeneous Networks (HetNets) consisting of macro cells and small cells sharing the same frequency band. Dense deployment of small cells overlaid by a macro layer is considered to be one of the most promising solutions for providing hotspot coverage in future 5G networks. The focus is to devise an optimised policy for small cells' access to the shared spectrum, in terms of their transmissions, in order to keep small cell served users sum data rate at high levels while ensuring that certain level of quality of service (QoS) for the macro cell users in the vicinity of small cells is provided. Both data and control channel constraints are considered, to ensure that not only the macro cell users' data rate demands are met, but also a certain level of Bit Error Rate (BER) is ensured for the control channel information. Control channel reliability is especially important as it holds key information to successfully decode the data channel. The problem is addressed by our proposed linear binary integer programming heuristic algorithm which maximises the small cells utility while ensuring the macro users imposed constraints. To further reduce the computational complexity, we propose a progressive interference aware low complexity heuristic solution. Discussion is also presented for the implementation possibility of our proposed algorithms in a practical network. The performance of both the proposed algorithms is compared with the conventional Reuse-1 scheme under different fading conditions and small cell loads. Results show a negligible drop in small cell performance for our proposed schemes, as a trade-off for ensuring all macro users data rate demands, while Reuse-1 scheme can even lead up to $40 \%$ outage when control region of the small cells in heavily loaded.
\end{abstract}

\section{Index Terms}

5G, Heterogeneous Networks, Small Cells, Inter-cell Interference, Resource Allocation, Binary Integer Linear Programming. 


\section{INTRODUCTION}

Heterogeneous Networks (HetNets) comprising macro cells and densely deployed small cells, are considered as a promising solution for future 5G networks [1]. It is indicated in [2], that dense deployment of Small Cell Access Points (SCAPs) $)^{1}$ can provide higher spectral efficiency, as compared to WiFi offloading. However, mass deployment of small cells overlayed within the area of larger cells raises challenges regarding their joint operation. SCAPs can usually operate in two modes; Open Subscriber Group (OSG) and Closed Subscriber Group (CSG). OSG SCAPs are deployed and owned by the network operator and operate as open cells to serve macrocell users in Hotspots or near the edges of the cells. This type of SCAPs are simple to manage and have demonstrated to improve access network capacity [3]. CSG SCAPs are typically owned by the subscriber and are open only to a long term managed list of users. On the other hand, CSG SCAPs are easy to manage if they are operated in a separate licence free band similar to Wi-Fi. However, these SCAPs, serving indoor subscribers as part of the operators network, need to be operated in a licensed band. Since the licensed spectrum resources are expensive and scarce, operators prefer to deploy these SCAPs under the so-called co-channel deployment, i.e. by spatially reusing the available spectrum. As a trade-off, this sharing of the frequency band amongst the macrocell and CSG SCAPs increases Inter-cell Interference (ICI) within the network which, if left unmanaged, may significantly deteriorate overall network performance [4]. ICI does not only effect the data channel, in fact also has a severe impact on the control channel reliability in heterogeneous networks. The control channel reliability is especially important as it contains key information to successfully decode the data channel. Hence, a poor control channel performance has a direct impact on the data channel performance of the network. This highlights the need for introduction of efficient low-complexity radio resource management techniques which not only maximise the opportunistic data rate performance of HetNets but also ensure the reliability in the control region, while keeping the complexity at a minimum level for real time implementation in practical systems.

\section{A. Related Work}

ICI has been widely discussed in literature, with focus initially targeted at homogeneous ${ }^{2}$ macrocell scenarios. The simplest downlink frequency allocation technique is to share the whole available frequency band amongst multiple transmission nodes. This so-called Reuse-1 technique has the highest spectrum usage but also results in severe ICI experienced amongst the neighbouring cells. To reduce ICI, Fractional Frequency Reuse (FFR) schemes were initially introduced [5]. However FFR schemes reduce the spectrum usage and are mostly preplanned in nature, prohibiting adaptive frequency allocation to system dynamics. More recently, Dynamic Fractional Frequency Reuse (D-FFR) techniques have been introduced. However, research works in [6]-[10] focus only on homogeneous macro cell scenarios. But since these techniques are only suitable for larger cells, they cannot perfectly fit to networks with underlaid macrocells and overlaid densely deployed small cells; the reason is that the dominant interferers for a user in the homogeneous scenario are limited and usually not as strong as in the dense HetNet scenario. Thus, focusing

\footnotetext{
${ }^{1}$ We interchangeably use the terms small cell and SCAP in this work.

${ }^{2}$ By homogeneous networks, we indicate the networks with same size and same access technology cells.
} 
on the HetNet scenario and on the SCAP-SCAP interference, [11] suggests that SCAPs should serve their users on sub-carriers with the least measured pilot signal strength from neighbouring SCAPs (hence the least SCAP-SCAP interference). Similarly, [12] proposes a technique where SCAPs assign the top best RBs to their users and adjust their transmit power subject to SCAP served users QoS constraints.

Although SCAP-SCAP interference is a notable aspect in HetNet scenario, the degradation of performance for macrocell served users due to interference caused from SCAPs to macrocell users will be more critical than in case of SCAP users; since there are fewer users served by SCAPs as compared to macrocells, SCAP served users are anyway allocated with more bandwidth resources. Thus, regarding the interference from SCAPs to macrocell users, [13] presents a bandwidth partitioning amongst macrocells and SCAPs, where SCAPs are not allowed to transmit in the bandwidth assigned to a macrocell, hence, reducing the spatial reuse. In [14], authors elaborate on the presence of CSG SCAPs further elevating the issue of ICI as compared to public SCAPs and discuss the use of shared, separate and partially shared bandwidth for this case. Furthermore [15] suggests the use of higher level modulation and coding schemes for indoor small cells as their users generally realise good signal strengths. In [16], a scheme is proposed which zones SCAP served users for either link adoption or requirement of orthogonal sub-bands and a central entity assigns the users with separate sub-bands from a pool. In [17], a mathematical framework is presented to minimise the interference from SCAPs to macrocells. SCAPs are allowed to transmit on certain sub-carriers based on the calculated distance between the SCAPs and neighbouring macrocells.

Since the objective of this research is to not only cope with ICI in the data channel as well as in the control channel, the above discussed literature only address the ICI in the data channel which can not be easily applied in the control channel, since it is not flexible enough to support ICI [18]. Evolved Inter Cell Interference Coordination (eICIC) is a proposed framework by $3^{\text {rd }}$ Generation Partnership Project (3GPP) to mainly focus on handling control channel interference in HetNet environments. [19] proposes an eICIC solution for control region assignment in Long Term Evolution-Advanced (LTE-A) networks with several aggregated carriers in a macro and small cells environment. The small cells control signalling is restricted in certain component carriers (CC), based on indicators form the neighbouring macrocells. While carrier aggregation (CA) based solutions are attractive for situations with large availability of spectrum and UEs with CA capability, non-CA (i.e., co-channel) based solutions are important to enable efficient heterogeneous network deployments with small bandwidth availability and legacy UEs without CA capability. [18] proposes a scheme for homogeneous Long Term Evolution (LTE) networks for reducing the control channel interference by minimising the transmit power and aggregation level allocated to downlink control channel.

LTE standards provide certain level of sparseness to reduce ICI in the control region, since the placement of control channels is based on a hash function of the physical cell identity (PCI) of each node [20]. Some researches have also exploited this property of LTE control channel to manage the PCI assignment minimising the control channel conflict probability [21]. This PCI optimisation technique is proven to be effective in larger cell networks, however due to limited number of PCIs and perfect protection only from fewer neighbouring cells makes this solution non feasible for small cell networks. Nevertheless, [22] proposes a solution to protect a specific channel in the control region (Physical Control Format Indicator (PCFICH)) based on PCI manipulation, where each SCAP 
detects the PCI of the strongest neighbouring macrocell and manipulates its own PCI to minimise the interference. However, we believe that for enhanced performance, SCAP muting decisions should be more adaptive to the system dynamics and consider the presence of macrocell served users in the vicinity of SCAPs. To the best of our knowledge there is no such analysis in literature based on the idea of interference estimation, caused by SCAPs to macrocell users. This is a notable shortcoming, as macro victim users trapped in the vicinity of CSG SCAPs suffer from severe interference [14].

\section{B. Contributions and Overview of the Paper}

In this paper, we investigate the dynamic resource allocation problem for OFDMA heterogeneous networks considering macro and small cells inter-tier interference. The aim of this work is to ensure a certain level of reliability for the macro users' control channel performance and also to meet a rate constraint in the data channel. These two constraints along with our objective to maximise the overall throughput of SCAP served users are accomplished by managing the transmissions of high interference causing SCAPs. We achieve this aim by optimising the small cell transmission on the control and data channel individually. It is important not only to optimise the system for the data channel but also for the control channel as there is vital information in the control channel which has certain reliability requirements. If the control channel is lost, the user is unable to decode the intended data channel as well. A dynamic heuristic scheme based on linear binary integer programming is proposed, which maximises the accumulative SCAP data rate and ensures macrocell users constraints. Furthermore, to reduce the complexity load in a practical network, we proposed a low complexity algorithm which only focuses on keeping the macro user faced interference below the estimated threshold by allowing the least interfering SCAPs in a progressively incremental order. In addition, we discuss in detail how our proposed heuristic schemes can be implemented in an existing OFDMA-based practical system such as LTE networks. Using Monte-Carlo simulations, it is demonstrated that our proposed schemes can ensure the macro users constraints as compared to Reuse-1 scheme while also keeping the SCAP users data rate at high levels. We consider SCAPs with variable load on the control channel and also vary the fading conditions to analyse and compare the performance of our proposed algorithms with the conventional Reuse-1 scheme.

The rest of this paper is organised as follows. Section II presents the system model along with mathematical formulation of the optimisation problem. Section III discusses the proposed heuristic schemes along with their pseudocodes. While Section IV sheds light on the feasibility for the practical implementation of the proposed schemes in LTE networks. Numerical results and obtained insights are discussed in Section V. Finally, Section VI concludes the paper. 


\section{System Model}

We consider a system of $M+1$ cells, comprising one macro cell (identified as cell 0 ) and $M$ small cells within the macro cell area (depicted in Figure 1). The set of small cells is defined as $\mathcal{M}=\{1, \ldots, M\}$. We assume that there are $K$ active users in the system. We consider that each user can have only one serving node, but each cell can support multiple users; thus, $K \triangleq|\mathcal{K}|=\left|\mathcal{K}_{0} \cup \mathcal{K}_{1} \cdots \cup \mathcal{K}_{M}\right|$, where $\mathcal{K}$ denotes the set of all users in the system and $\mathcal{K}_{m}$ denotes the set of users served by node in cell $m$. A Resource Element (RE) represents the smallest unit of physical resource and a Resource Block (RB) which is made up of a number of REs in frequency and time domain, represents the smallest transmission unit in downlink of an OFDMA system. $\mathcal{T}_{v}$ represents the set of all $T_{v}$ REs in a RB, and $\mathcal{T}_{(n)}$ represents the set of all $T_{(n)}$ REs allocated to a user for control information regarding RB $n$. And, super-set $\mathcal{T}$ represents all $\mathcal{T}_{(n)}$ sets: $\mathcal{T}=\left\{\mathcal{T}_{(1)}, \ldots, \mathcal{T}_{(n)}, \ldots\right\}$. We consider a minimal level of reliability (minimum required BER) on the control channel of the macro cell and small cell served users, which is vital for the successful decoding of the data channel. Furthermore, for the macro cell users we consider a minimum required rate on the data channel. For this study, we consider only the dedicated user-specific control channel information to be protected. SCAP nodes reuse the same resources as of the macro cell to serve their small-cell users (SUEs) based on a resource allocation policy. We consider a central entity which is able to collect relevant information to make resource allocation decisions and guide small cells on the resource allocation policy to be adopted. In the following subsections we express the SINR and interference allowance representations for the control and data channels of MUEs and SUEs. It is important to represent control and data channel parameters separately as the constraints on the control channel are different from the data channel. The power levels on the control channel are different from the data channel. In addition, the data channel is capable of higher modulation and coding schemes, however the control channel is usually transmitted at the most robust modulation and coding scheme.

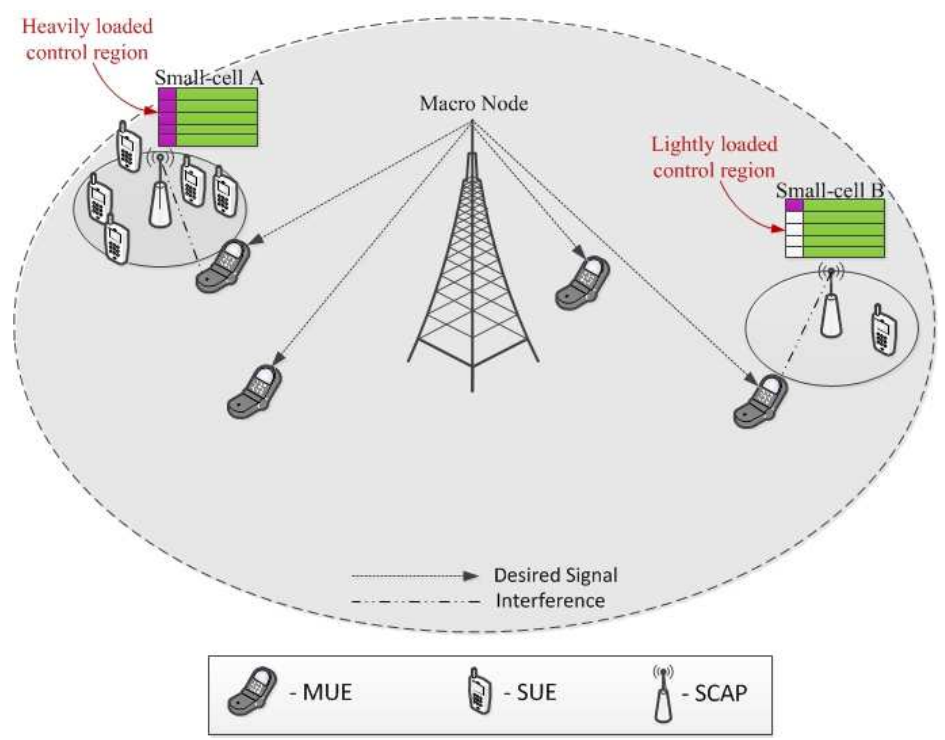

Fig. 1: System Model 


\section{A. Control Channel: User SINR \& Max Interference Allowance}

The SINR of MUE $u$ at RE $t \in \mathcal{T}_{n}$, bearing control information for MUE $u$ is given by

$$
\gamma_{u, 0, t}^{\mathrm{c}}=\frac{p_{0, n}^{\mathrm{c}} \Gamma_{u, 0, n}^{0}}{\sum_{m=1}^{M}\left(\sum_{k \in \mathcal{K}_{m}} \phi_{k, m, t}^{\mathrm{c}}\right) p_{m, n}^{\mathrm{c}} \Gamma_{u, 0, n}^{m}+N_{0} B_{t}},
$$

where $p_{0, n}^{\mathrm{c}}$ denotes the transmit power of macro cell node at $\operatorname{RE} t, \Gamma_{k, m, n}^{i}$ is the channel gain between base station at cell $i$ and user $k$ being served at cell $m$ in RB $n, N_{0}$ is the noise power spectral density and $B_{t}$ is the bandwidth of each RE. And $\phi_{k, m, t}^{\mathrm{c}} \in\{0,1\}$, where $\phi_{k, m, t}^{\mathrm{c}}=1$ when $\mathrm{RE} t$ is assigned to bear control information for $k^{\text {th }}$ user in small cell $m$; otherwise, the RE allocation parameters take zero value. $\phi^{\mathfrak{c}}=\left[\phi_{1,1,1}^{\mathfrak{c}} \ldots \phi_{K_{M}, M, T_{t}}^{\mathrm{c}}\right]$, where $T_{t}$ is the total number of REs used for control in TTI $t$. Similarly SINR of SUE $k$ in cell $m$ at RE $t$ is expressed as

$$
\gamma_{k, m, t}^{\mathrm{c}}=\frac{p_{m, n}^{\mathrm{c}} \Gamma_{k, m, n}^{m}}{p_{0, n}^{\mathrm{c}} \Gamma_{k, m, n}^{0}+\sum_{\substack{i=1 \\ i \neq m}}^{M}\left(\sum_{l \in \mathcal{K}_{i}} \phi_{l, i, t}^{\mathrm{c}}\right) p_{i, n}^{\mathrm{c}} \Gamma_{k, m, n}^{i}+N_{0} B_{t}} .
$$

where transmit power of the SCAP node at $m^{\text {th }}$ cell at each RE in the control-section of $n^{\text {th }}$ RB is denoted by $p_{m, n}^{\mathrm{c}} \leq P_{\max }$.

The fixed MUE demand BER at RE can be translated into a specific minimum required $\gamma_{u, 0, t}^{\text {c-req }}$ SINR value, given a certain aggregation level (e.g. CCE aggregation level in LTE) used for the specific user. Maximum interference power $\Omega_{u, t}^{\mathrm{c}-\max }$ that MUE $u$ can tolerate in RE $t$ from all SCAP nodes to be able to decode its UE-specific control information correctly is given by

$$
\Omega_{u, t}^{\mathrm{c}-\max }=\frac{p_{0, n}^{\mathrm{c}} \Gamma_{u, 0, n}^{0}}{\gamma_{u, 0, t}^{\mathrm{c}-\mathrm{req}}}-N_{0} B_{t}
$$

If the potential channel gain from any small cell $m$ to the MUE at $\operatorname{RE} t$ is denoted as $\Gamma_{0, u, n}^{m}$, the total interference caused to it by all small cells in this RE can be given by

$$
\Omega_{u, t}^{\mathrm{c}-\text { sum }}=\sum_{m=1}^{M}\left(\sum_{k \in \mathcal{K}_{m}} \phi_{k, m, t}^{\mathrm{c}}\right) p_{m, n}^{\mathrm{c}} \Gamma_{0, u, n}^{m}=\sum_{m=1}^{M}\left(\sum_{k \in \mathcal{K}_{m}} \phi_{k, m, t}^{\mathrm{c}}\right) \omega_{0, u, t}^{m} .
$$

where $\omega_{0, u, t}^{m} \triangleq p_{m, n}^{\mathrm{c}} \Gamma_{u, 0, n}^{m}$ can be interpreted as the interference that is caused to user $u$ in cell 0 (macro cell) on RE $t$ from small cell $m$.

Similarly, the fixed SUE demand BER at RE $t$ can be translated into a specific minimum required $\gamma_{k, m, t}^{\mathrm{c} \text { req }}$ SINR value. This SINR value is used to estimate the Maximum interference, $\Omega_{k, m, t}^{c-m a x}$ that is required to decode the UEspecific control information, given by

$$
\Omega_{k, m, t}^{\mathrm{c}-\max }=\frac{p_{m, n}^{\mathrm{c}} \Gamma_{k, m, n}^{m}}{\gamma_{k, m, t}^{\mathrm{c}-\mathrm{req}}}-N_{0} B_{t} .
$$

The total interference at RE $t$ experienced by SUE $k$ from macro cell and neighbouring SCAPs transmissions is given by

$$
\Omega_{k, m, t}^{\mathrm{c}-\mathrm{sum}}=p_{0, n}^{\mathrm{c}} \Gamma_{k, m, n}^{0}+\sum_{\substack{i=1 \\ i \neq m}}^{M}\left(\sum_{l \in \mathcal{K}_{i}} \phi_{l, i, t}^{\mathrm{c}}\right) p_{i, n}^{\mathrm{c}} \Gamma_{k, m, n}^{i}
$$




\section{B. Data Channel: User SINR \& Max Interference Allowance}

The SINR of MUE $u$ served in RB $n$ is given by

$$
\gamma_{u, 0, n}^{\mathrm{d}}=\frac{p_{0, n}^{\mathrm{d}} \Gamma_{u, 0, n}^{0}}{\sum_{m=1}^{M}\left(\sum_{k \in \mathcal{K}_{m}} \phi_{k, m, n}^{\mathrm{d}}\right) p_{m, n}^{\mathrm{d}} \Gamma_{u, 0, n}^{m}+N_{0} B_{n}},
$$

where $p_{0, n}^{\mathrm{d}}$ denotes the transmit power of macro cell node at $\mathrm{RB} n, \Gamma_{k, m, n}^{i}$ is the channel gain between base station at cell $i$ and user $k$ being served at cell $m$ in $\mathrm{RB} n, N_{0}$ is the noise power spectral density and $B_{n}$ is the bandwidth of each RB. $\phi_{k, m, n}^{\mathrm{d}} \in\{0,1\}$, where $\phi_{k, m, n}^{\mathrm{d}}=1$ when small cell $m$ serves its $k^{\text {th }}$ assigned user in the $n^{\text {th }} \mathrm{RB}$; otherwise, the RB allocation parameters take the zero value. $\phi^{\mathrm{d}}=\left[\phi_{1,1,1}^{\mathrm{d}} \ldots \phi_{K_{M}, M, N}^{\mathrm{d}}\right]$. Similarly the SINR of SUE $k$ in cell $m$ at $\mathrm{RB} n$ is given by

$$
\gamma_{k, m, n}^{\mathrm{d}}=\frac{\phi_{k, m, n}^{\mathrm{d}} p_{m, n}^{\mathrm{d}} \Gamma_{k, m, n}^{m}}{p_{0, n}^{\mathrm{d}} \Gamma_{k, m, n}^{0}+\sum_{\substack{i=1 \\ i \neq m}}^{M}\left(\sum_{l \in \mathcal{K}_{i}} \phi_{l, i, n}^{\mathrm{d}}\right) p_{i, n}^{\mathrm{d}} \Gamma_{k, m, n}^{i}+N_{0} B_{n}}
$$

where transmit power of the small cell node at $m^{\text {th }}$ cell in the data-section of $n^{\text {th }} \mathrm{RB}$ is denoted by $p_{m, n}^{\mathrm{d}} \leq P_{\max }$. The data channel SINR expressed in (8) is different from the control channel SINR expressed in (2), since the power levels on the control channel differ from those in the data channel. Secondly the data channel SINR is expressed on the RB level whereas for the control channel it is expressed on the RE level.

The User Data Rate (SUE or MUE) at RB $n$ can be expressed as follows

$$
R_{k, m, n}=\log _{2}\left(1+\gamma_{k, m, n}^{\mathrm{d}}\right)=\log _{2}\left(1+\phi_{k, m, n}^{\mathrm{d}} p_{m, n}^{\mathrm{d}} \alpha_{k, m, n}^{\mathrm{d}}\right),
$$

where $\alpha_{k, m, n}^{\mathrm{d}}$ denotes the channel gain of the user $k$ served by cell $m$ at RB $n$.

The fixed MUE demand data rate at RB $n$ can be translated into a specific minimum required $\gamma_{u, 0, n}^{\text {d-req }}$ SINR value (using equation (9)). Maximum interference power $\Omega_{u, n}^{\max }$ that MUE $u$ can tolerate in RB $n$ from all SCAP nodes to obtain this rate threshold is given by:

$$
\Omega_{u, n}^{\mathrm{d}-\max }=\frac{p_{0, n}^{\mathrm{d}} \Gamma_{u, 0, n}^{0}}{\gamma_{u, 0, n}^{\mathrm{d}-\mathrm{req}}}-N_{0} B_{n} .
$$

If the potential channel gain from any small cell $m$ to the MUE at RB $n$ is denoted as $\Gamma_{0, u, n}^{m}$, the total interference caused to it by all small cells in this RB can be given by:

$$
\Omega_{u, n}^{\mathrm{d}-\mathrm{sum}}=\sum_{m=1}^{M}\left(\sum_{k \in \mathcal{K}_{m}} \phi_{k, m, n}^{\mathrm{d}}\right) p_{m, n}^{\mathrm{d}} \Gamma_{0, u, n}^{m}=\sum_{m=1}^{M}\left(\sum_{k \in \mathcal{K}_{m}} \phi_{k, m, n}^{\mathrm{d}}\right) \omega_{0, u, n}^{m},
$$

where $\omega_{0, u, n}^{m} \triangleq p_{m, n}^{\mathrm{d}} \Gamma_{u, 0, n}^{m}$ can be interpreted as the interference that is caused to user $u$ in cell 0 (macro cell) on RB $n$ from small cell $m$. 


\section{Optimisation Problem}

Finally, the overall optimisation problem is formulated as follows:

$$
\max _{\mathbf{p}^{\mathrm{d}}, \mathbf{p}^{\mathrm{c}}, \boldsymbol{\phi}^{\mathrm{d}}, \boldsymbol{\phi}^{\mathrm{c}}, \mathcal{T}} \sum_{n=1}^{N} \sum_{m=1}^{M} \sum_{k \in \mathcal{K}_{m}}\left(\prod_{t \in \mathcal{T}_{(n)}} c_{k, m, t}\left(\mathbf{p}^{\mathrm{c}}, \boldsymbol{\phi}^{\mathrm{c}}\right)\right) R_{k, m, n}\left(\mathbf{p}^{\mathrm{d}}, \boldsymbol{\phi}^{\mathrm{d}}\right)
$$

subject to:

$$
\begin{array}{r}
\phi_{k, m, n}^{\mathrm{d}} \in\{0,1\}, \forall k \in \mathcal{K} \backslash \mathcal{K}_{0}, m \in \mathcal{M}, n ; \\
\sum_{k \in \mathcal{K}_{m}} \phi_{k, m, n}^{\mathrm{d}} \leq 1, \forall m \in \mathcal{M}, n ; \\
\phi_{k, m, t}^{\mathrm{c}} \in\{0,1\}, \forall k \in \mathcal{K} \backslash \mathcal{K}_{0}, m \in \mathcal{M}, t ; \\
\sum_{k \in \mathcal{K}_{m}} \phi_{k, m, t}^{\mathrm{c}} \leq 1, \forall m \in \mathcal{M}, t ; \\
\Omega_{n}^{\mathrm{d}-\mathrm{sum}} \leq \Omega_{n}^{\mathrm{d}-\max }, \quad \forall n ; \\
\Omega_{u, t}^{\mathrm{c}-\mathrm{sum}} \leq \Omega_{u, t}^{\mathrm{c}-\max }, \\
\sum_{n=1}^{N}\left(\sum_{k \in \mathcal{K}_{m}} \phi_{k, m, n}^{\mathrm{d}}\right) p_{m, n}^{\mathrm{d}} \leq P_{\max }, \forall m \in \mathcal{M} ; \\
\sum_{n=1}^{\mathrm{d}} \geq 0, \forall m \in \mathcal{M}, n ; \\
\sum_{t \in \mathcal{T}_{n}}\left(\sum_{k \in \mathcal{K}_{m}} \phi_{k, m, t}^{\mathrm{c}}\right) p_{m, n}^{\mathrm{c}} \leq P_{\max }, \forall m \in \mathcal{M} ; \\
p_{m, n}^{\mathrm{c}} \geq 0, \forall m \in \mathcal{M}, n,
\end{array}
$$

where,

$$
c_{k, m, t}= \begin{cases}1, & \text { if } \Omega_{k, m, t}^{\mathrm{c}-\mathrm{sum}} \leq \Omega_{k, m, t}^{\mathrm{c}-\max } \\ 0, & \text { otherwise. }\end{cases}
$$

The main maximisation objective is expressed in (12) and regarding the sum data rates for the small cell users $c_{k, m, t}$ is elaborated in (14) being a binary multiplier to ensure that if the control channel information is not decodable in a certain instance the data channel is also lost, since the control channel holds vital information to decode the data channel. Constraints in (13a) and (13c) express the binary nature of the $\phi$ muting variable for data and control channel respectively. Constraints (13b) and (13d) guarantee that $\mathrm{RB} n$ in data region and $\mathrm{RE} t$ in control region is assigned to at most one user $k$. Maximum interference tolerance constraints for the data and control channel of MUE are expressed in (13e) and (13f) respectively. Constraints (13g) and (13h), and (13i) and (13j) together fulfil the power constraints for the data channel and control channel respectively. It is important to mention here that we do not impose any minimum quality constraints on control and data channels of SUEs. Usually small cells have as much resources as the larger marcocell. However, due to restriction on maximum power, they cover a very small area, resulting in relatively very few users in their area of coverage. Hence, the small cells have enough resources to serve their SUEs. The formulated optimisation problem being very complex in nature is extremely difficult to 
solve in real network with dynamically changing environment. To address the complexity issues we devise two low complexity heuristic solutions, explained in the following section.

\section{Proposed Resource Allocation Schemes}

The proposed Small Cell Rate Maximisation (SRM) and Interference Tolerance Aware (ITA) resource allocation schemes will be presented in this section. The purpose of these schemes is to heuristically achieve the objective expressed in the optimisation problem in (12), and also keeping computational complexity at a very low level. The main difference between the SRM and ITA scheme is that the SRM scheme solves the same maximisation problem by binary integer programming with relaxation on the transmit power (transmit power is kept fixed). However, in case of ITA the focus is to allow SCAP transmissions considering minimum caused interference to the MUE in a progressively incremental order till the constrained tolerance threshold is reached.

\section{A. Small Cell Rate Maximisation Resource Allocation (SRM)}

The main objective of the proposed SRM resource allocation scheme is to maximise the overall small cells data rate and along with that to satisfy the macro user constraints on the control and data channels. This is achieved by finding the optimal combination of SCAP transmissions restriction matrix using binary integer programming. The proposed algorithm first finds SCAPs control channel restriction matrix $\overrightarrow{\phi_{t}^{c}}$ followed by the data channel restriction

matrix $\overrightarrow{\phi_{n}^{\mathrm{d}}}$. For the control channel restriction matrix $\overrightarrow{\phi_{t}^{\mathrm{c}}}$, initially the maximum interference allowance $\Omega_{u, t}^{\mathrm{c}-\max }$ is estimated for every RE $t$ in each RB as expressed in equation (3). Next, for every SCAP $m$, the interference it causes to MUE being served on that RE $t$ is updated as expressed in (4). Similarly, the data channel expected rate for the every SUE in every SCAP $m$ is estimated. The maximum interference allowance, the estimated interference from each SCAP to macro cell user and the estimated achievable rate of SCAPs are used to solve the binary integer problem. This is estimated for each RE to create and update the SCAPs control channel restriction matrix. Once the control channel restriction matrix is updated and notified to the respective SCAPs, the restriction matrix for the data channel is solved for each RB. The binary integer problem for the data channel is solved similarly to the control channel but on the RB level. The maximum interference allowance $\Omega_{u, n}^{\mathrm{d}-\max }$, the estimated interference from each SCAP to MUE $\vec{\omega}_{u, 0, n}$ and the estimated achievable rate of SCAPs $\overrightarrow{\hat{R}}$ are used to solve the data channel restriction matrix $\overrightarrow{\phi_{n}^{\mathrm{d}}}$. This restriction matrix is further notified to the SCAPs and remains valid until the next update is available. The steps in Algorithm 1 summarise the processes required for the SRM resource allocation scheme.

\section{B. Interference Tolerance Aware Resource Allocation (ITA)}

Since the SRM scheme solves the binary integer programming problem for each RE in the control channel, this scheme would still be quite computationally complex for implementation in a real network. Interference Tolerance Aware Resource Allocation (ITA) is a modified version of SRM scheme, the motivation behind this scheme is to minimise the control channel computational complexity for more feasible implementation of this scheme in a practical network. Considering the granularity of the required optimisation on each RE in the control region, this ITA 


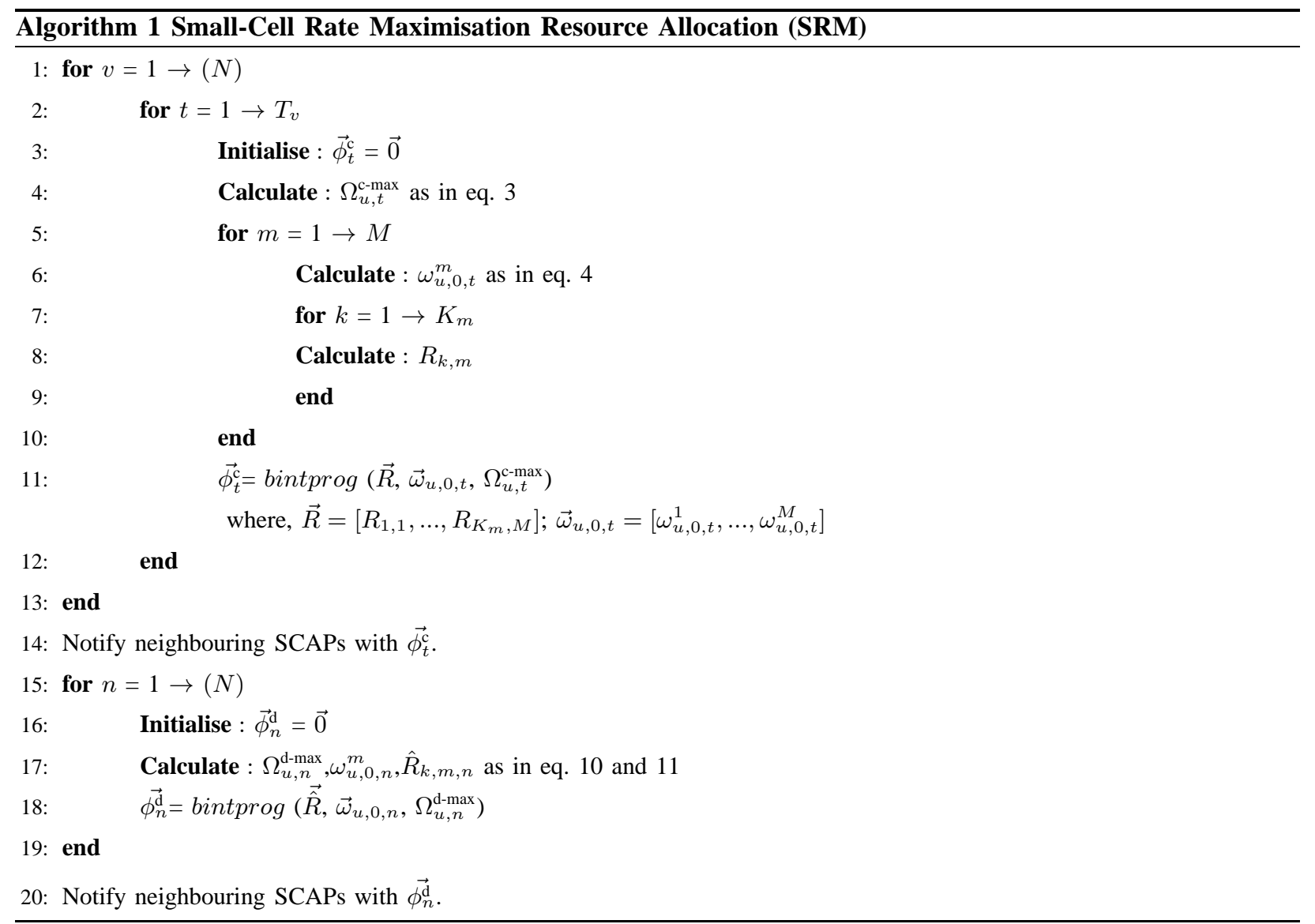

scheme overrides the binary optimisation problem with a simple sorting policy. Instead of maximising the SCAPs overall data rate, the scheme only focuses on creating the SCAP transmissions' restriction matrix by allowing the control channel transmissions of SCAPs' with the least caused interference $\omega_{u, 0, t}^{m}$ towards MUEs.

For the control channel restriction matrix, initially the maximum interference allowance $\Omega_{u, t}^{c-m a x}$ is estimated for every RE $t$ in each RB as expressed in equation (3). Next, the interference caused by each SCAP towards MUE being served on the specific RE is estimated. The channel gain $\Gamma_{k, m, t}^{m}$ of each SUE is also estimated. In the following stage, the estimated interference $\omega_{u, 0, t}^{m}$ from each SCAP to MUE is sorted in an ascending list $\vec{\omega}_{u, 0, t}^{\text {sort }}$. The elements of $\vec{\omega}_{u, 0, t}^{s o r t}$ are added to $\Omega_{u, t}^{\mathrm{c} \text {-sum }}$ in each iteration and are compared against the maximum interference allowance $\Omega_{u, t}^{\mathrm{c}-\text { max }}$. For each iteration, if the magnitude of $\Omega_{u, t}^{\text {c-sum }}$ remain less than maximum interference allowance, the SCAP on top of the $\vec{\omega}_{u, 0, t}^{s o r t}$ is allowed to transmit on that specific RE. Preference is given to SUE served by SCAP with highest channel gain in each iteration. If no more control channel REs are required for the SUE with highest channel gain, the next SUE is given preference. The loop breaks when the allocation reaches the maximum interference tolerance limit. The resulting control channel restriction matrix $\overrightarrow{\phi_{t}^{c}}$ is notified to the respective SCAPs. The data channel restriction matrix $\overrightarrow{\phi_{n}^{\mathrm{d}}}$ is generated in a similar way as in the SRM algorithm. The steps in Algorithm 2 summarise the processes required for the ITA resource allocation scheme. 


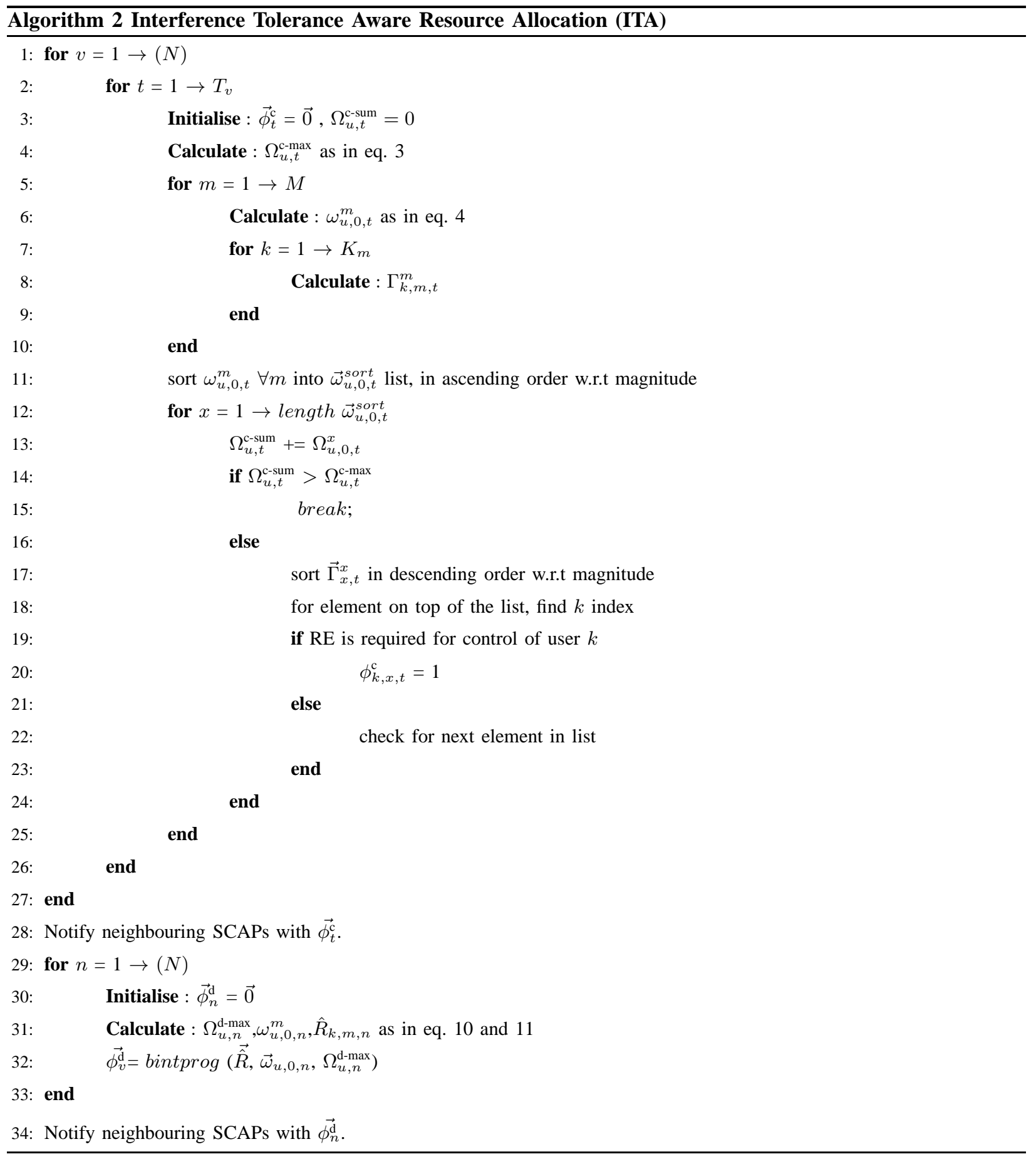




\section{IMPLEMENTATION IN LTE NETWORKS}

In this section, a high level description is provided on how the proposed resource allocation schemes can be implemented in LTE heterogeneous networks comprising macro and small cells.

\section{A. LTE Frame Structure and Control Channels}

In LTE OFDMA - frequency division duplex (FDD) the whole system bandwidth is divided into RBs of $180 \mathrm{KHz}$ each. Each RB consists of 12 sub-carriers (15KHz each), however, a RB represents the basic OFDMA time-frequency unit. A Physical Resource Block (PRB) consists for one RB in the frequency domain and two constitutive slots in the time domain, where each slot is $0.5 \mathrm{msec}$. These two consecutive time slots make one subframe (1msec) and ten such subframes make one 10ms radio frame. Each PRB consists of 14 OFDM symbols (12 in extended mode), of which first three symbols are usually considered for common and user specific control signalling (control region is dynamic from 1 to 3 symbols). The remaining symbols constitute of the data channels. Inter-spread within these are cell specific reference symbols, which help facilitate channel estimation. The most important control channels are discussed below. The detailed LTE resource allocation grid is given in Figure. 2.

The main three LTE control channels are 1) physical hybrid-ARQ indicator channel (PHICH), 2) physical control format indicator channel (PCFICH) and, 3) physical downlink control channel (PDCCH). The PHICH contains the uplink hybrid-ARQ ACK/NACK information, indicating weather the serving cell has correctly received an uplink transmission from the user and the PHICH location in the grid is cyclically rotated dependent on the PCI of that cell and is three times redundantly repeated in the frequency domain. It can occur on any combination of the available OFDM symbols, however usually present in the first OFDM symbol (presence in the second and third OFDM symbol is possible under the extended PHICH configuration). The PCFICH is always present in the first OFDM symbol only; since it contains the control format indicator (CFI) indicating the number of OFDM symbols used for transmission of downlink control channel; the reason being simple, user should be able to determine the size of the control region before decoding the remaining control information. Two bits of information is sufficient to indicate the CFI information (1, 2 or 3 OFDM symbols), however, this vital information is made redundant by a 32 bits long corresponding code word which amount to a code rate of 1/16. This 32 bit long information is mapped to 16 REs using quadrature phase-shift keying (QPSK) modulation. The PCFICH is transmitted in groups of four REs, distributed four times in the frequency domain to achieve frequency diversity. The PCFICH location in the grid is cyclically rotated dependent on the PCI of the cell, similar to the PHICH. PHICH and PCFICH can be partially protected by manipulating the PCI of the small cells in accordance with marco cells PCI as proposed in [22]. However, the macro cell transmitted PDCCH to the MUE still remains unguarded against the interference from the small cells.

The PDCCH contains the downlink (and uplink) scheduling grants, power control commands and the information required to decode and demodulate the OFDM symbols in the downlink (encode and modulate in the uplink). The PDCCH dedicated to a specific user may consist of 1,2,4,8 control channel elements (CCEs), depending on the selected aggregation level based on the prevailing channel conditions between the serving cell and the user. Link adaptation is performed considering the vital information contained in the PDCCH, a target BER of $1 \%$ is pursued. 
Each CCE is made up of nine resource element groups (REGs) and each REG consists of four consecutive resource elements (REs). The REGs still free after placement of PHICH and PCFICH are allocated to PDCCH after going through a inter-column permutation interleaving pattern [23]. Thus, our proposed algorithms aim to protect the user specific PDCCH data for the macrocell UE, maintaining a certain level of acceptable BER.

\section{B. Implementation feasibility of the proposed scheme in LTE}

The following arguments explain how the key functions and elements of LTE architecture can be used for this reason.

A: UEs report their CQI and demand rate to their serving cells on frequent basis. Based on these reports received from MUEs, equations (9) and (10) can be used to estimate the maximum interference, $\Omega_{n}^{d-\text { max }}$, that a MUE can tolerate on a certain RB. Similarly, given a target BER for control region, the required minimum SINR can be calculated to estimate the maximum interference $\Omega_{n}^{c-\max }$, using equations (3).

B: UEs also report to their serving cell, the neighbouring cell's Reference Signal Received Quality (RSRQ) along with the PCI of the neighbouring cell. These reports are generally used for A2, A3 and A4 measurements based handovers. In our case, the respective MUE reports can be used to estimate the top neighbouring interfering small cells; then, this information can be used to estimate the total interference caused to it by all small cells in each RE/RB, $\Omega_{n}^{d-\max } / \Omega_{t}^{c-\max }$, and formulates the optimisation constraints in (13e) and (13f).

C: Moreover, the addition of X2 logical interface in LTE provides the means for cells to communicate. Amongst the macro cell and the neighbouring small cells, X2 can act as an interface to guide the neighbouring small cells to restrict their transmissions in the control and data region. Thus, X2 interface can be used to input each small cell utility (i.e. expected rate of SUEs in the small cell based on equation (9)) at each RB to the central entity at the macro cell. The input from all small cells, formulates our objective function in (12) (i.e. maximisation of expected sum rate of all SUEs in the system).

D: Finally, the optimisation process for either of the proposed algorithms is performed at the central entity. The optimisation function returns $\phi_{k, m, t}^{c}$ and $\phi_{k, m, n}^{d}$. These parameters are passed to small cells over the X2 interface and act as restriction matrix of each small cell for the control and data channel respectively. 


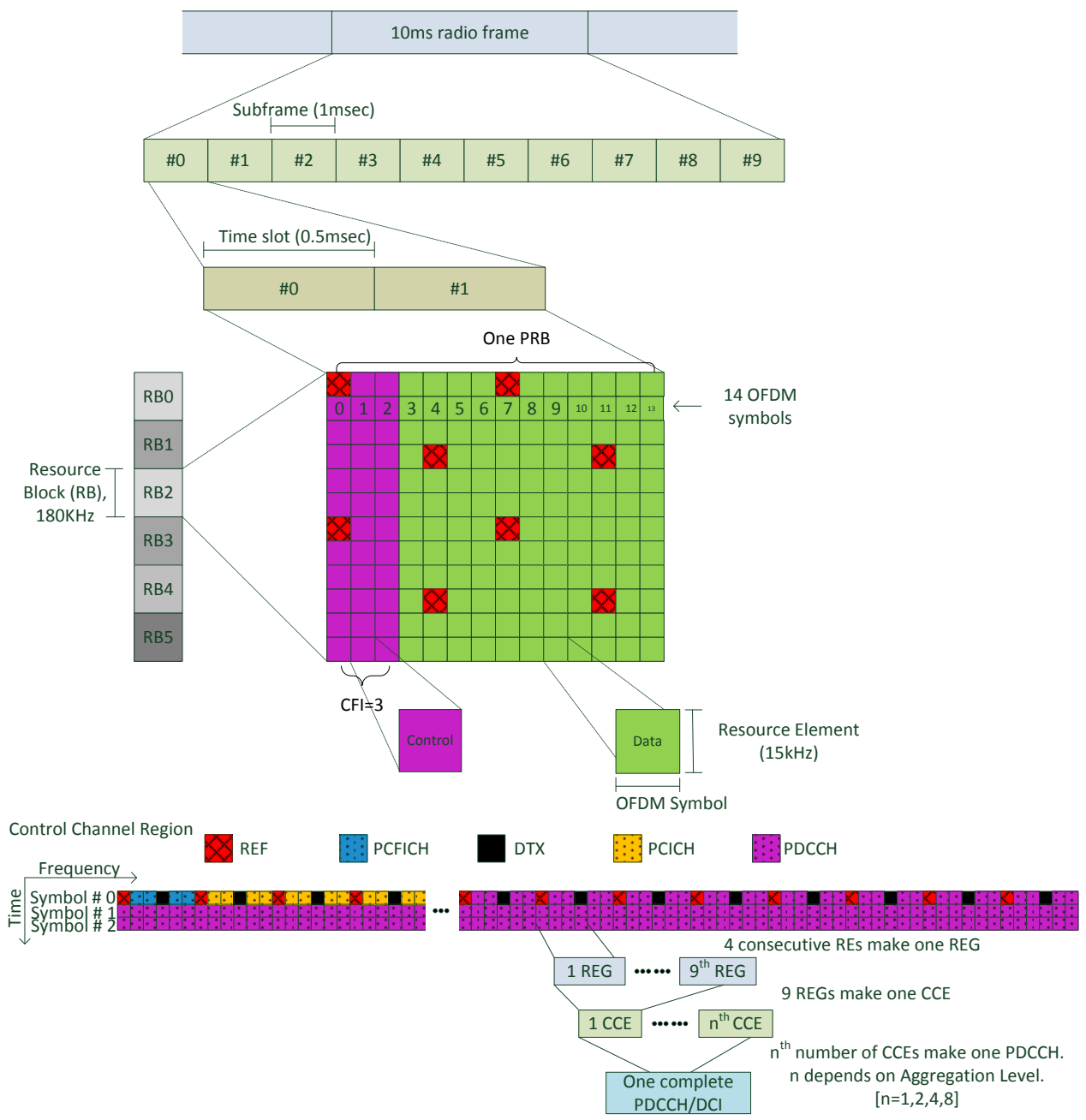

Fig. 2: LTE Resource Allocation Grid

\section{NumericAl RESUlts}

In this section we show the numerical results for our proposed schemes and compare them to the conventional Reuse-1 scheme. For this numerical analysis we simulate a single macro cell along with five small cells. The macro cell and each SCAP is serving upto five SUEs. We consider shadowing and fast fading in our simulation. Proposed schemes are also analysed for variations in shadow fading environments, shadow fading effects are incorporated into path-loss estimated by addition of a zero-mean Gaussian random variable, with standard deviation $\sigma$. For these simulations the MUE minimum required data rate was set to $1.1 \mathrm{Mbps}$. Further details of the simulation parameters are given in Table I.

The Cumulative Distribution Function plots in Figure 3 and 6 represent the macro users data rates for the case when the SCAPs are lightly loaded and heavily loaded respectively. We consider the control region of SCAP to be lightly loaded if less than $20 \%$ of the control is occupied and heavily loaded if more than $80 \%$ of the control region is occupied. We observe that for the Reuse-1 case as the shadow fading standard deviation coefficient is increased, the number of MUEs in outage increase. In case of lightly loaded SCAPs (in Figure 3), Reuse-1 scheme has an 
TABLE I: LTE-Based Scenario - Simulation Parameters.

\begin{tabular}{|l|c|c|}
\hline Parameter & Macro-cell & Small-cell \\
\hline Number of nodes & 1 & 5 \\
\hline Carrier frequency & \multicolumn{2}{|c|}{$2.1 \mathrm{GHz}$} \\
\hline Bandwidth & \multicolumn{2}{|c|}{$5 \mathrm{MHz}$} \\
\hline Node transmit power & $43 \mathrm{dBm}$ & $23 \mathrm{dBm}$ \\
\hline Path loss model & \multicolumn{2}{|c|}{$128.1+37.6 \log _{10}(d[\mathrm{Km}])$} \\
\hline Number of UEs & 5 & $1-5 \mathrm{UE}$ per SCAP \\
\hline Number of OFDM symbols for PDCCH & 3 & 3 \\
\hline BER threshold for PDCCH & \multicolumn{2}{|c|}{18} \\
\hline Number of RE Quadruplets per PDCCH & \multicolumn{2}{|c|}{$9 \mathrm{~dB}$} \\
\hline Noise Figure at UE & \multicolumn{2}{|c|}{$-174 \mathrm{dBm} / \mathrm{Hz}$} \\
\hline Thermal noise density & \multicolumn{2}{|c|}{$50 \mathrm{~m}$} \\
\hline Cell Radius & \multicolumn{2}{|c|}{3} \\
\hline
\end{tabular}

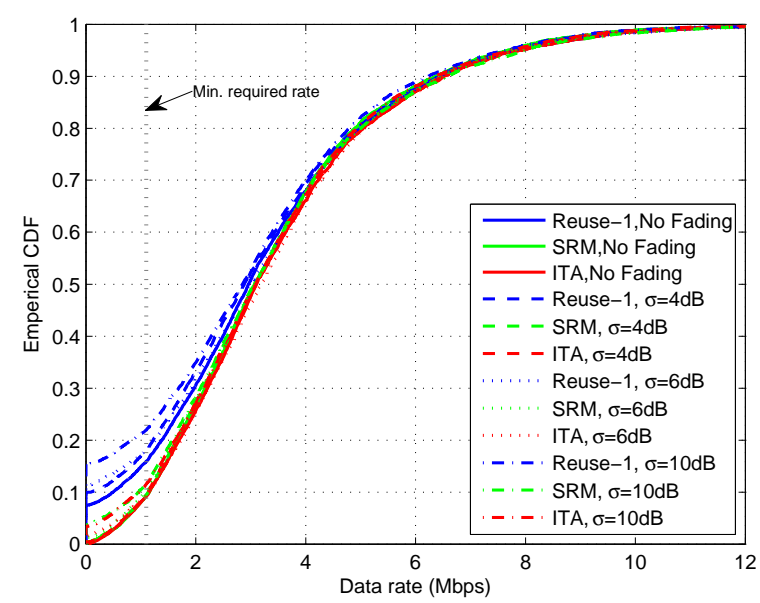

Fig. 3: CDF plot for MUE data rates for the lightly loaded SCAPs case.

outage of nearly $10 \%$ users and as the shadow fading co-efficient is increased to $\sigma=10 \mathrm{~dB}$, outage reaches upto $20 \%$. In case of SRM and ITA the outage is kept very low as compared to Reuse-1 case. We also add a fading margin to our algorithms which can be updated based on preceding channel conditions. Similarly in case of heavily loaded SCAPs (in Figure 6, Reuse-1 has a very poor performance, outage MUEs reach upto $45 \%$ and even further as the shadow fading co-efficient is increase. The efficiency of both the proposed algorithms can be seen in Figure 6, where the outage MUEs are kept below $15 \%$ even in high fading environments. These results can be further analysed with the help of the Box and Whisker plots in Figure 4 and 7, indicating the range of MUE data rates between 9\%ile and 91\%ile and also indicating the first and third quartiles of the data. For lightly loaded SCAPs (Figure 7) and shadow fading $\sigma=10 d B$, SRM and ITA manage to keep the MUE outage below 10\%ile, whereas for Reuse-1 the outage reaches close to the first quartile (25\%ile). Similarly in the heavily loaded SCAPs case and shadow fading $\sigma=10 \mathrm{~dB}$, SRM and ITA keep the MUE outage below the 25\%ile, whereas for Reuse-1 the outage is above the $50 \%$ ile. 


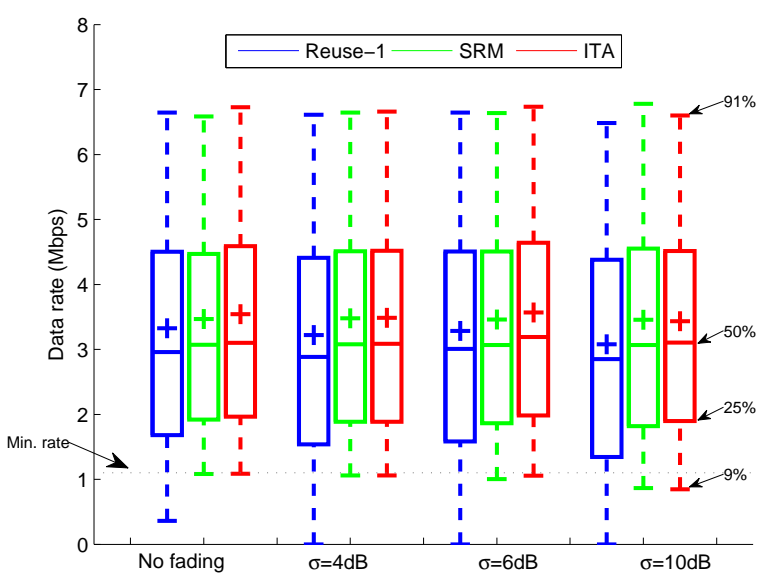

Fig. 4: Box and Whiskers plot for MUE Data rates for lightly loaded SCAPs case. Each plot ranges from 9\%ile to $91 \%$ ile of the values where as the box expresses the lower and upper quartile (25\% and $75 \%$ ). Line dividing the box expresses the median and ' + ' expresses the mean value.

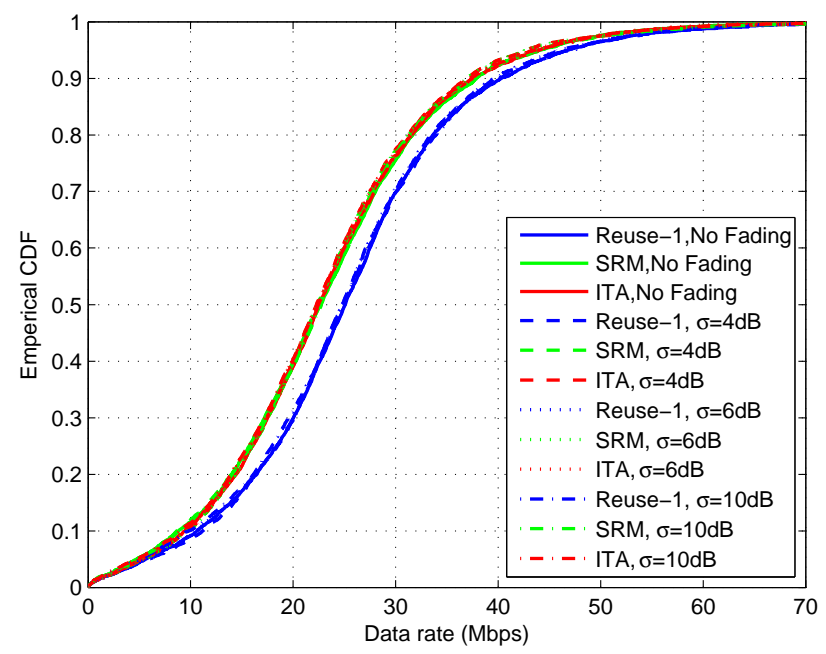

Fig. 5: CDF plot for SCAP individual data rates for the lightly loaded SCAPs case.

The gains for the proposed algorithms come as a trade-off to the data rates at the SCAPs. In Figure 5 and 8 the SCAPs individual throughputs are presented, for lightly loaded SCAPs and heavily loaded SCAPs case respectively. It is evident that the Reuse-1 case has the maximum SCAPs throughputs, nevertheless SRM and ITA schemes have comparable throughputs with negligible degradation. We must mention here that considering the fact that a SCAP usually serves fewer users as compared to a macro cell, slight degradation in the SCAP's overall throughput does not severally effects its users due to ample amount of available resources. Also to point out that in terms of SCAP throughputs, ITA lags slightly behind SRM which comes as a trade-off to the minimalistic computational complexity of ITA scheme. 


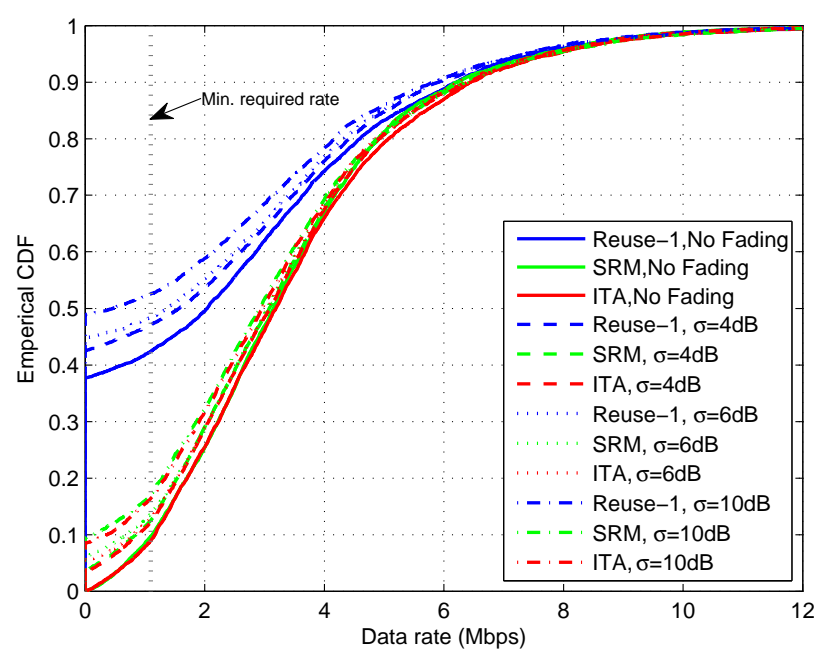

Fig. 6: CDF plot for MUE data rates for the heavily loaded SCAPs case.

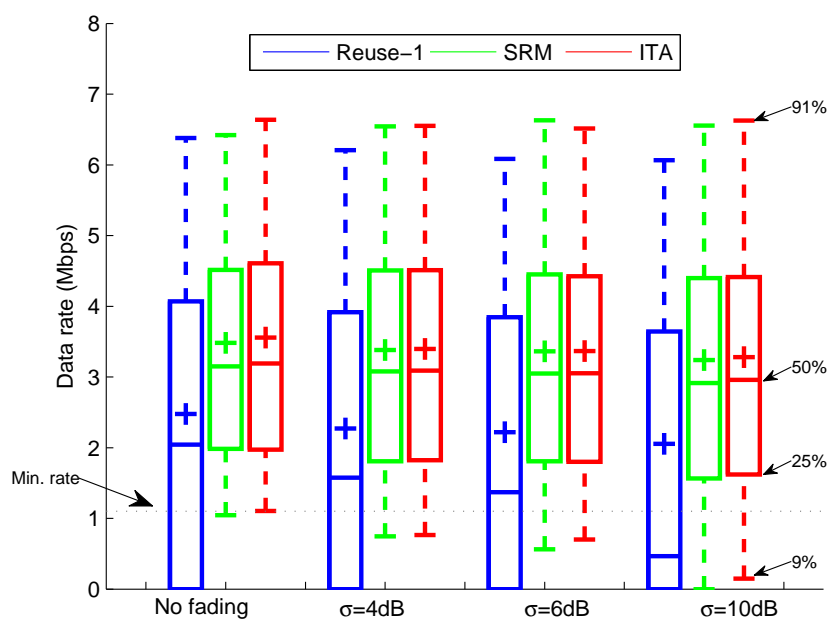

Fig. 7: Box and Whiskers plot for MUE Data rates for heavily loaded SCAPs case.

In Figure 9 and 10, we present the percentage loss of PDCCH in the MUEs for various shadow fading environments. It is evident from the bar graphs that if the SCAPs are heavily loaded there are severe PDCCH losses, reaching nearly upto 50\% in certain high shadow fading conditions for Reuse-1 case. Our proposed algorithms manage to keep the PDCCH losses relatively low, hence protecting macro cell users from outage.

It is also interesting to see how the shadow fading margin in our algorithms plays an important role in protecting macro users control region. The plots in Figure 11,12,13 show our simulations for a fixed shadow fading standard deviation of $\sigma=6 \mathrm{~dB}$, and the margin is varied from $3 \mathrm{~dB}$ upto $10 \mathrm{~dB}$ to analyse the performance under extreme fading conditions. A larger margin obviously means more MUE control region protection, but also means that in return a lower SCAPs throughput. It is clear that even with lower margin our proposed ITA scheme performs better than 


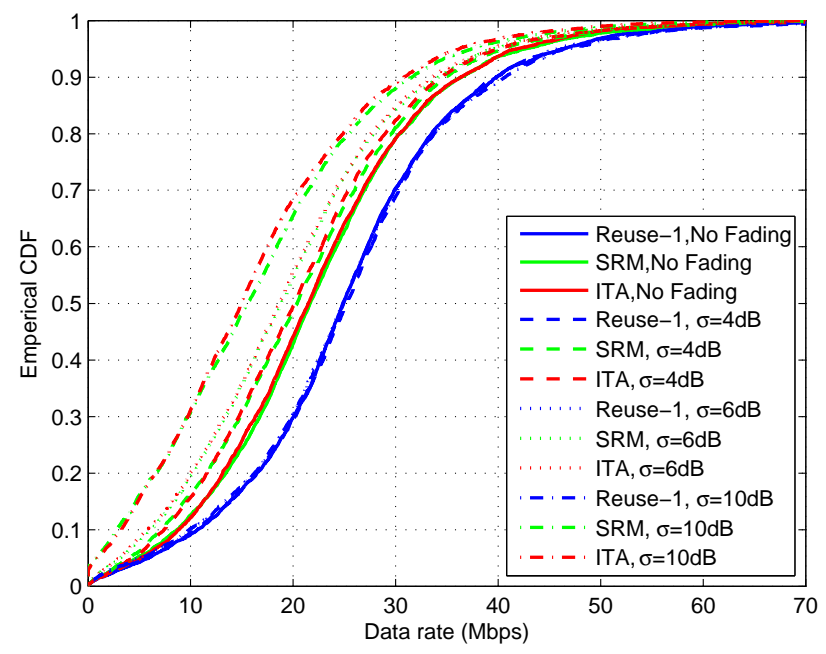

Fig. 8: CDF plot for SCAP individual data rates for the heavily loaded SCAPs.

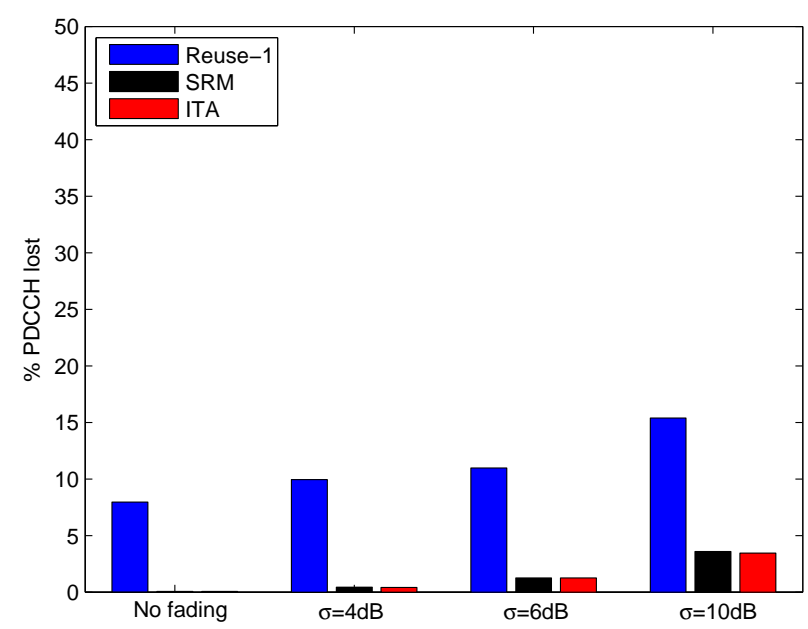

Fig. 9: Percentage lost PDCCH in lightly loaded SCAP case.

Reuse- 1 case, and has a very low computational complexity.

The complexity of the optimal case to solve the control channel resource allocation is $O\left((K M T)^{2}\right)$, where $K$ represents the number of UEs, $M$ represents the number of SCAPs and $T$ represents the number of REs. The complexity of the optimal solution in our case is quadratic in nature and is extremely infeasible to implement in a real network. The proposed SRM scheme is solved by binary linear integer programming. There are several linear programming relaxations applied to such algorithms, which make them very effective in practice but it is difficult to prove the theoretical complexity bounds on the performance of such algorithms. A comparison in terms of normalised CPU time is presented for SRM, ITA and the optimal case in Figure 14. It is evident that optimal case has a very high complexity and is not feasible to solve in a practice system. On the other hand SRM and ITA 


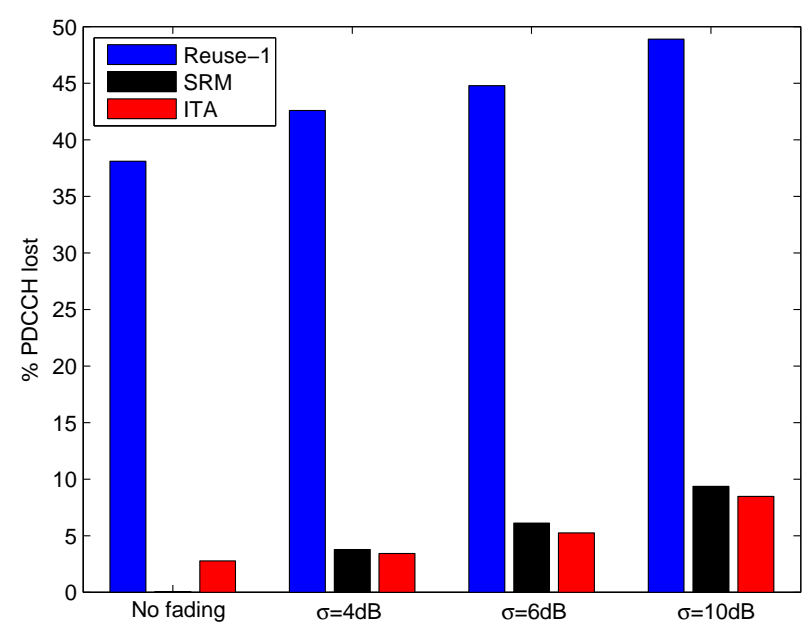

Fig. 10: Percentage lost PDCCH in heavily loaded SCAP case.

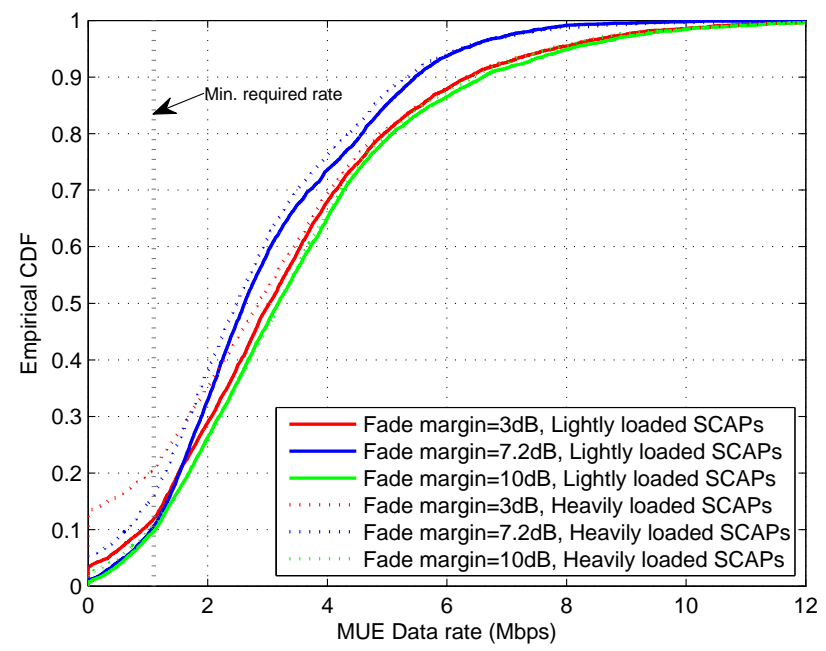

Fig. 11: CDF of MUE Data rates (ITA scheme) for various fading margins.

schemes have a significantly lower complexity. In fact ITA scheme has very minimal complexity and poses to be a very favourable candidate to be applied in a practical network. 


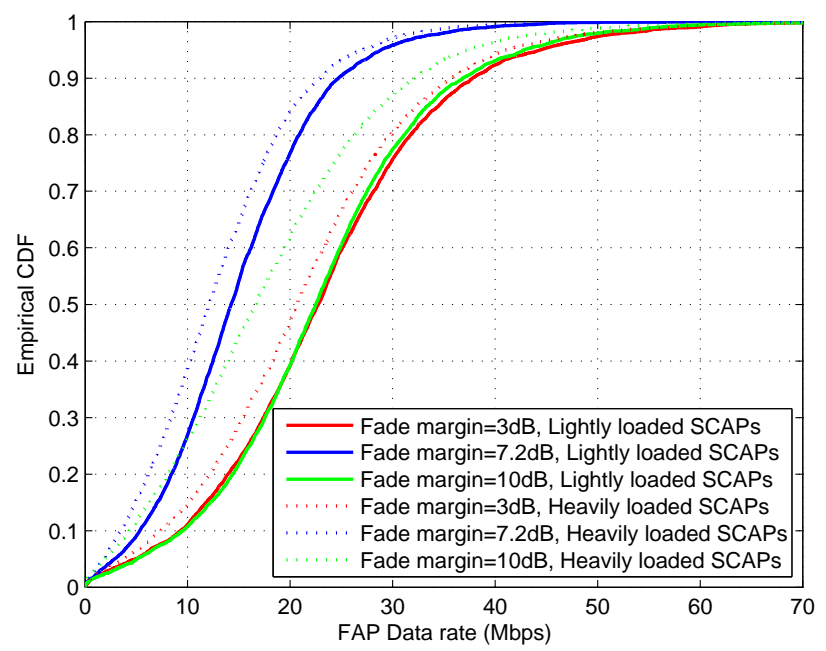

Fig. 12: CDF of SCAPs individual Data rates (ITA scheme) for various fading margins.

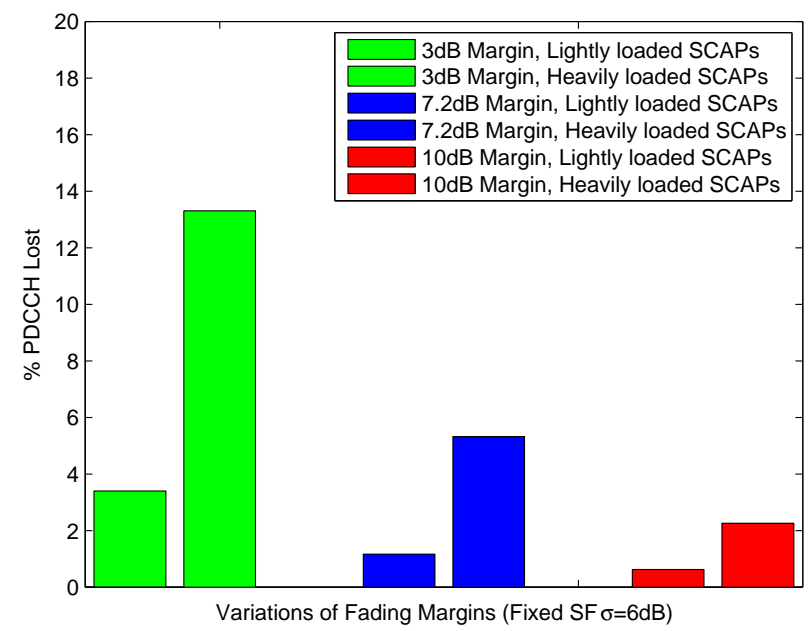

Fig. 13: Percentage PDCCH lost (ITA scheme).

\section{Conclusions}

Inter-tier interference deteriorates the performance of mobile macro cell served users in a HetNet environment comprising macro and small cells sharing the same frequency band. With most existing studies focusing on increasing the performance of small cell users, macro-cell users demands have been usually out of the equation. However, especially in the upcoming $5 \mathrm{G}$ networks envisaging the dense deployment of small cells overlaid by a macro umbrella, inter-tier interference levels will see a huge increase affecting significantly this type of users. In this paper, we examined ways to manage inter-tier interference in OFDMA-based networks though coordinated multicell resource allocation. Coordinated resource allocation is considered not only for the data channel but also for the control channel whose reliability is especially important as it contains key information to successfully decode the data 


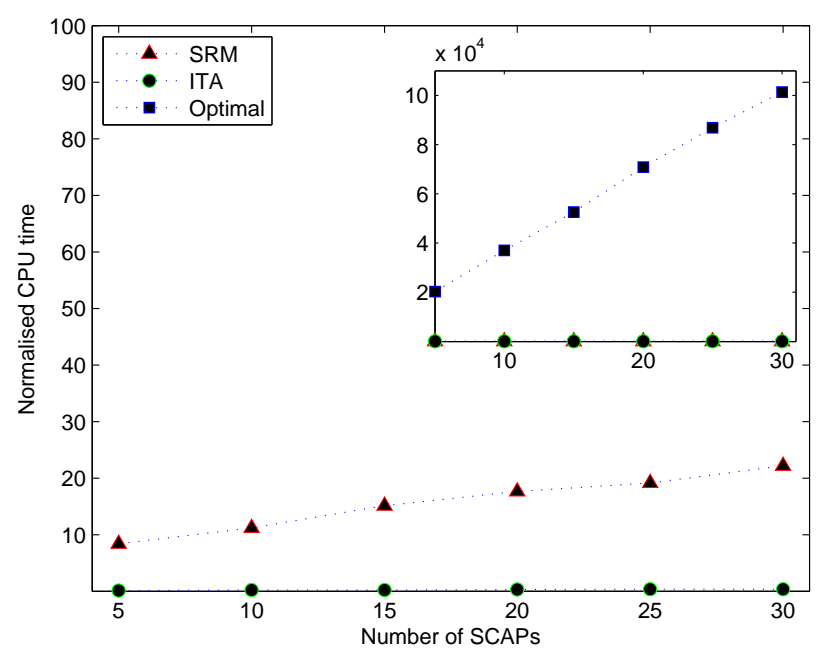

Fig. 14: Computational complexity comparison

channel. To this end, a performance optimisation problem for this novel scenario is mathematically formulated. We propose two dynamic resource allocation algorithms at small cells to maximize their sum data rate while at the same time the interference faced by the macro cell served users is kept below a tolerance threshold. Interference tolerance is estimated based on macro users minimum rate requirement at the data channel and minimum BER threshold to decode the control information at the control channel. Our simulation results demonstrate that the proposed Small-cell Rate Maximisation (SRM) resource allocation scheme ensures the macro users QoS requirements while keeping the small cell users data rates similar as compared to the conventional Reuse- 1 scheme. To reduce the computational complexity we propose a low complexity Interference Tolerance Aware (ITA) resource allocation scheme. This scheme ensures nearly similar performance compared to SRM scheme. Further we also discuss the practical implementation of our proposed scheme in LTE networks. As 5G networks are expected to be even more flexible for control and data resource allocation, this implementation can serve as a guide on how to perform coordinated resource allocation in future systems for achieving high performances ubiquitously over the network.

\section{ACKNOWLEDGEMENT}

We would like to acknowledge the support of the University of Surrey 5GIC (http://www.surrey.ac.uk/5gic) members for this work. 


\section{REFERENCES}

[1] V. Chandrasekhar, J. Andrews, and A. Gatherer, "Femtocell Networks: a Survey," IEEE Communications Magazine, vol. 46, no. 9, pp. 59-67, 2008

[2] I. Hwang, B. Song, and S. Soliman, “A Holistic View on Hyper-dense Heterogeneous and Small Cell Networks,” IEEE Communications Magazine, vol. 51, no. 6, pp. 20-27, June 2013.

[3] S. Navaratnarajah, A. Saeed, M. Dianati, and M. Imran, "Energy Efficiency in Heterogeneous Wireless Access Networks," IEEE Wireless Communications Magazine, vol. 20, no. 5, pp. 37-43, October 2013.

[4] A. Saeed, A. Akbari, M. Dianati, and M. A. Imran, "Energy Efficiency Analysis for LTE Macro-Femto HetNets," in Proceedings of 19th European Wireless Conference (EW), April 2013.

[5] S.-E. Elayoubi and B. Fourestie, "On Frequency Allocation in 3G LTE Systems," in Proceedings of IEEE 17th International Symposium on Personal, Indoor and Mobile Radio Communications (PIMRC), Sept 2006.

[6] S. Ali and V. C. M. Leung, "Dynamic Frequency Allocation in Fractional Frequency Reused OFDMA Networks," IEEE Transactions on Wireless Communications, vol. 8, no. 8, pp. 4286-4295, August 2009.

[7] R. Y. Chang, Z. Tao, J. Zhang, and C.-C. J. Kuo, "A Graph Approach to Dynamic Fractional Frequency Reuse (FFR) in Multi-cell OFDMA Networks," in Proceedings of IEEE International Conference on Communications, 2009, pp. 3993-3998.

[8] A. Stolyar and H. Viswanathan, "Self-Organizing Dynamic Fractional Frequency Reuse in OFDMA Systems," in Proceedings of IEEE 27th Conference on Computer Communications (INFOCOM), April 2008.

[9] D. Lopez-Perez, C. Xiaoli, and Z. Jie, "Dynamic Downlink Frequency and Power Allocation in OFDMA Cellular Networks," IEEE Transactions on Communications, vol. 60, no. 10, pp. 2904-2914, 2012.

[10] D. Lopez-Perez, X. Chu, A. V. Vasilakos, and H. Claussen, "Power Minimization Based Resource Allocation for Interference Mitigation in OFDMA Femtocell Networks," IEEE Journal on Selected Areas in Communications, vol. 32, no. 2, pp. 333-344, February 2014.

[11] J. Ling, D. Chizhik, and R. Valenzuela, "On Resource Allocation in Dense Femto-Deployments," in Proceedings of IEEE International Conference on Microwaves, Communications, Antennas and Electronics Systems, Nov 2009.

[12] F. Bernardo, R. Agusti, J. Cordero, and C. Crespo, "Self-Optimization of Spectrum Assignment and Transmission Power in OFDMA Femtocells," in Proceedings of Sixth Advanced International Conference on Telecommunications (AICT), May 2010.

[13] V. Chandrasekhar and J. Andrews, "Spectrum Allocation in Tiered Cellular Networks," IEEE Transactions on Communications, vol. 57, no. 10, pp. 3059-3068, October 2009.

[14] J. D. Hobby and H. Claussen, "Deployment Options for Femtocells and Their Impact on Existing Macrocellular Networks," Bell Labs Technical Journal, vol. 13, no. 4, pp. 145-160, 2009.

[15] H. Claussen, "Co-Channel Operation of Macro- and Femtocells in a Hierarchical Cell Structure," International Journal of Wireless Information Networks, vol. 15, no. 3-4, pp. 137-147, 2008

[16] M. Arslan, J. Yoon, K. Sundaresan, S. Krishnamurthy, and S. Banerjee, "A Resource Management System for Interference Mitigation in Enterprise OFDMA Femtocells," IEEE/ACM Transactions on Networking, vol. 21, no. 5, pp. 1447-1460, Oct 2013.

[17] X. Chu, Y. Wu, D. Lopez-Perez, and X. Tao, "On Providing Downlink Services in Collocated Spectrum-Sharing Macro and Femto Networks," IEEE Transactions on Wireless Communications, vol. 10, no. 12, pp. 4306-4315, December 2011.

[18] D. Gonzalez G, M. Garcia-Lozano, and S. Ruiz Boque, "Power Allocation for the PDCCH in LTE: A Way to Increase Its Capacity in Realistic Deployments," in Wireless Personal Multimedia Communications (WPMC), 2013 16th International Symposium on, June 2013.

[19] S. Uygungelen, Z. Bharucha, and H. Taoka, "Control Region Protection in LTE-A Networks," in Personal Indoor and Mobile Radio Communications (PIMRC), 2012 IEEE 23rd International Symposium on, Sept 2012, pp. 986-991.

[20] Z. Bharucha, G. Auer, and T. Abe, "Downlink Femto-to-Macro Control Channel Interference for LTE," in Wireless Communications and Networking Conference (WCNC), 2011 IEEE, March 2011, pp. 1259-1264.

[21] H. Yang, A. Huang, R. Gao, T. Chang, and L. Xie, "Interference Self-Coordination: A Proposal to Enhance Reliability of System-Level Information in OFDM-Based Mobile Networks via PCI Planning," Wireless Communications, IEEE Transactions on, vol. 13, no. 4, pp. 1874-1887, April 2014.

[22] Z. Bharucha, G. Auer, T. Abe, and N. Miki, "Femto-to-macro control channel interference mitigation via cell id manipulation in lte," in Vehicular Technology Conference (VTC Fall), 2011 IEEE, Sept 2011, pp. 1-6.

[23] 3GPP TS36.212, "Evolved Universal Terrestrial Radio Access (E-UTRA); Multiplexing and Channel Coding (Release 9)," 2010. 